\title{
Lideranças Tóxica e Empoderadora: Estudo de Validação de Medidas em Amostra Portuguesa
}

\author{
Toxic and Empowering Leadership: Measures Validation Study in a Portuguese \\ Sample
}

\author{
Lisete Mónico ${ }^{1}$, Ana Salvador ${ }^{2}$, Nuno Rebelo dos Santos ${ }^{3}$, Leonor Pais ${ }^{4}$ e Carla Semedo ${ }^{5}$
}

\begin{abstract}
Resumo
O presente trabalho tem como propósito proceder à validação recíproca para a língua portuguesa da Toxic Leadership Scale e do Empowering Leadership Questionnaire. O conceito de empoderamento e o conceito de toxicidade na liderança são conceptualmente opostos, refletindo, respetivamente, efeitos detrimentais e desenvolvimentais nos colaboradores. Após os procedimentos técnicos de tradução (e retroversão) dos itens dos dois instrumentos, ambos foram aplicados a uma amostra de 408 trabalhadores portugueses de diversos setores de atividade. As análises fatoriais confirmatórias indicam um bom ajustamento às estruturas fatoriais originais dos dois instrumentos de medida. Os índices de precisão (precisão compósita e Alpha de Cronbach) mostram-se adequados. As correlações negativas obtidas entre as dimensões das medidas de liderança tóxica e de liderança empoderadora corroboram a oposição conceptual entre ambos os constructos. O estudo realizado fornece evidência empírica da validade das medidas de liderança tóxica e de liderança empoderadora, constituindo um referencial para a validação noutros países lusófonos.
\end{abstract}

Palavras-chave: liderança, liderança empoderadora, liderança tóxica, Empowering Leadership Questionnaire, Toxic Leadership Scale

\begin{abstract}
The purpose of this paper is to validate for the Portuguese language the Toxic Leadership Scale and the Empowering Leadership Questionnaire. The concept of empowerment and the concept of leadership toxicity are conceptually opposite reflecting, respectively, detrimental and developmental effects on subordinates. After the technical procedures of translation (and back translation) of the items of the two instruments, both were applied to a sample of 408 Portuguese workers from several activity sectors. Confirmatory factor analysis indicate a good fit to the original factorial structures of the two instruments. The reliability (composite reliability and Cronbach's Alpha) is adequate. The negative correlations obtained between the dimensions of toxic leadership and empowering leadership support the conceptual opposition between both constructs. This study provides empirical evidence of the validity of the measures of toxic leadership and empowerment leadership, being a reference for validation in other Portuguese-speaking countries.
\end{abstract}

Keywords: leadership, toxic leadership, empowering leadership, Toxic Leadership Scale, Empowering Leadership Questionnaire

\footnotetext{
${ }^{1}$ Doutorada em Psicologia Social, Professora Auxiliar na Faculdade de Psicologia e Ciências da Educação, Universidade de Coimbra, Rua do Colégio Novo, S/N, 3000-115 Coimbra, Portugal. Tel: +351913476965. E-mail: lisete.monico@fpce.uc.pt

${ }^{2}$ Mestre em Psicologia na Escola de Ciências Sociais, Universidade de Évora, Largo dos Colegiais, 2, $7004-516$ Évora, Portugal. Tel: +351968619579. E-mail: anasalvador1234@gmail.com

${ }^{3}$ Doutorado em Psicologia, Professor Auxiliar na Escola de Ciências Sociais, Universidade de Évora, Departamento de Psicologia, Largo dos Colegiais, 2, 7004-516 Évora, Portugal. Tel: +351 964217152. E-mail: nrs@uevora.pt

${ }^{4}$ Doutorada em Psicologia do Trabalho e das Organizações, Professora Auxiliar na Faculdade de Psicologia e de Ciências da Educação, Universidade de Coimbra, Rua do Colégio Novo, S/N, 3000-115 Coimbra, Portugal. Tel: +351919276549. E-mail: leonorpais@fpce.uc.pt

${ }^{5}$ Doutorada em Psicologia, Professora Auxiliar na Escola de Ciências Sociais, Universidade de Évora, Departamento de Psicologia, Largo dos Colegiais, 2, 7004-516 Évora, Portugal. Tel: +351 967907705. E-mail: cssemedo@ uevora.pt

Revista Iberoamericana de Diagnóstico y Evaluación - e Avaliação Psicológica. RIDEP · No53 · Vol.4 · 129-140 · 2019

ISSN: 1135-3848 print /2183-6051online
} 


\section{Introdução}

Os líderes, pela posição que ocupam, são facilmente percebidos como modelos, tendo, portanto, um impacto naqueles que estão sob a sua liderança e na sociedade em geral. Esse impacto vai além da eficácia e da eficiência na persecução dos objetivos organizacionais. Tanto a liderança empoderadora como a liderança tóxica contêm esta suposição implícita, visto que as respetivas definições vão para além da eficiência e eficácia.

As motivações para o exercício da liderança são diferenciadas, e provavelmente influenciam a forma com esta é exercida (Solano \& Minervino, 2007). No entanto, o foco do presente trabalho é na validação e análise da precisão de medidas que avaliam a forma como a liderança é exercida nos liderados. Mais concretamente, pretende-se analisar as propriedades psicométricas de dois instrumentos de avaliação da liderança, uma com efeito desenvolvimental (empoderadora) e outra com consequências detrimentais nos liderados (tóxica).

A liderança (enquanto exercício de poder) é aqui definida, numa adaptação da proposta de Smircich e Morgan (1982), como um processo de influência social na persecução de objetivos validados pelo conjunto dos indivíduos com quem aquele que exerce a liderança tem uma relação hierárquica estabelecida. Efetivamente, existe uma aceitação por parte dos liderados, no mínimo tácita, relativamente ao que são os objetivos partilhados que orientam a ação coletiva, que no mínimo é suficiente para que se mantenham na situação. Essa influência exerce-se, em primeiro lugar, sobre eles, isto é, aqueles que se encontram hierarquicamente abaixo (subordinados) e tem neles um inevitável impacto emocional e cognitivo, expresso no desempenho de cada um e na qualidade emocional da vivência no trabalho. Sabemos que existem distintas formas dos indivíduos lidarem com as suas emoções no trabalho (Salessi \& Omar, 2016). Por isso, embora seja expectável que diferentes respostas emocionais de diferentes indivíduos sejam dadas ao mesmo estilo de exercício do poder, é defensável que esses diferentes estilos sejam promotores de padrões específicos predominantes de resposta nos liderados.

Muitos foram os estudos sobre liderança nos últimos 100 anos. A abordagem funcionalista colocou ênfase na eficiência e na eficácia e observou-se a passagem de modelos universais a modelos contingenciais, bem como a passagem do foco em atributos de personalidade para o foco em comportamentos (Jago, 1982). Porém, a partir da década de 90, e como resposta à turbulência crescente na sociedade e no contexto das organizações, surgiram inúmeras soluções conceptuais salientando âncoras imunes à constante mudança. Essas respostas, no estudo da liderança, foram visíveis no surgimento de modelos virtuosos e positivos, por um lado, e contra virtuosos e negativos, por outro, os quais salientaram aquilo que, no exercício da liderança, contamina aqueles que se encontram em contacto com o líder, muito particularmente os subordinados ou seguidores (Bambale, 2014; Hansbrough \& Jones, 2014; Hernandez, Eberly, Avolio, \& Johnson, 2011; May, Wesche, Heinitz, \& Kerschreiter, 2014; Miniotaité \& Bučiūnienè, 2013; Steele, 2011). Essa contaminação, positiva ou negativa, tem componentes cognitivos e emocionais, e é expectável que se expresse, positiva ou negativamente, no desempenho e em outras variáveis que se consideram componentes da qualidade de vida e que, por isso, constituem valores em si mesmas.

Pelo seu conteúdo, esses modelos de liderança podem ser considerados vinculados a valores. $\mathrm{O}$ presente trabalho é focado em dois modelos conceptualmente antagónicos. Um deles é centrado na influência positiva do líder sobre os colaboradores, nomeadamente empoderando-os, a liderança empoderadora (Auh, Menguc, \& Jung, 2014), e o outro é centrado na influência negativa, a liderança tóxica (Steele, 2011).

Chen, Sharma, Edinger, Shapiro e Farh (2011) estudaram o efeito da liderança na motivação dos liderados, revelando que relações conflituosas têm efeito na "amotivação". Utilizando o Empowering Leadership Questionnaire (ELQ), Srivastava, Bartol e Locke (2006) obtiveram resultados que indicam que a liderança empoderadora está relacionada positivamente com a partilha de conhecimento e com a eficácia da equipa. Lorinkova, Pearsall e Sims (2013), utilizando a mesma escala, concluíram que a liderança empoderadora, comparativamente com a liderança diretiva, permite à equipa melhorar mais o seu desempenho. Contudo, trata-se de um processo 
que demora mais tempo, pois é um crescimento ao longo do tempo, que vai dando capacidade às equipas para se irem superando. Tuckey, Dollard e Bakker (2012) estudaram a liderança empoderadora enquanto inspiradora de envolvimento no trabalho, otimizando as condições do trabalho. Por último, Auh, Menguc e Jung (2014) constataram uma influência direta e positiva entre a liderança empoderadora e os comportamentos dos liderados.

Quanto à liderança tóxica, é definida pela literatura como um tipo de liderança mal ajustada e maliciosa (Whicker, 1996), em que os líderes têm um comportamento destrutivo e podem exibir certas características pessoais consideradas disfuncionais (Lipman-Blumen, 2005), maltratando as pessoas com quem trabalham, envenenando o seu entusiasmo, a sua criatividade, a sua autonomia e a sua capacidade de inovar (Wilson-Starks, 2003). No contexto gerado por estes líderes, é o seu estado de ânimo que determina o clima da organização (Flynn, 1999). Além disso, estes líderes manifestam uma falta de preocupação para com os seus subordinados, aspeto que afeta o clima organizacional, surgindo a convicção de que o líder apenas se motiva com assuntos do seu interesse pessoal (Reed, 2004).

A liderança tóxica tem elevado interesse para muitas organizações, quer militares (Di Genio, 2002; Reed, 2004; Williams, 2005) quer não militares (Kusy \& Halloway, 2009; Pelletier, 2010; Walton, 2007). Este interesse decorre das consequências que este tipo de liderança pode trazer. É sugerido que estas consequências impactam tanto na saúde dos empregados como na própria organização (Dyck, 2001), conduzindo a uma maior taxa de absentismo (Macklem, 2005) e rotatividade (turnover) (Flynn, 1999), baixo desempenho (Wilson-Starks, 2003) e consequente diminuição do volume de negócios (Flynn, 1999).

Por serem ambas conceptualmente focadas no efeito sobre os subordinados, a tarefa de validação psicométrica e contraste destes dois tipos de liderança, empoderadora e tóxica, ganha especial relevância, constituindo o foco do presente trabalho. Sendo uma focada no efeito positivo e desenvolvimental e a outra no efeito negativo e detrimental sobre os subordinados, espera-se que emerjam associações negativas entre ambas, suportando reciprocamente a existência de validade. Nesta sequência, formulamos a seguinte hipótese: as dimensões da liderança empoderadora correlacionam-se negativa e significativamente com as dimensões da liderança tóxica.

\section{Método}

\section{Participantes}

No presente estudo, recorreu-se a uma técnica de amostragem não probabilística de conveniência (Hill \& Hill, 2008). Foram elegíveis, como participantes, indivíduos trabalhadores em organizações a laborar em Portugal, de qualquer setor de atividade, tendo uma relação hierárquica com o atual superior há pelo menos três meses, e um igual período de tempo de emprego na atual entidade empregadora. A entidade empregadora deveria ter, no mínimo, nove colaboradores, já que organizações abaixo desse número facilmente têm sobreposição de papéis. O critério temporal decorreu de ter sido considerado necessário um tempo mínimo para que os trabalhadores pudessem pronunciar-se de forma fundamentada sobre as características das respetivas lideranças, assim como para terem clareza quanto à sua própria motivação para o trabalho.

A amostra inicial foi constituída por 411 participantes. Três foram excluídos por omissão de dados em vários itens das escalas, originando uma amostra efetiva de 408 participantes $(N=408)$, maioritariamente do sexo feminino $(57.1 \%, n=233)$ e trabalhadores do setor privado (69.1\%, n=282). A idade dos participantes distribui-se entre os 18 e os 76 anos, sendo a média de 39.8 anos $(M=398 ; D P=11.77)$. No que diz respeito às habilitações escolares, $38.0 \%$ dos respondentes indica ter terminado o ensino secundário, $1.5 \%$ refere saber ler e escrever e os restantes $34.1 \%$ possuem formação superior.

Entre os participantes do estudo, 76.2\% referem não ter um papel de chefia dentro da sua organização. No que concerne à dimensão da organização, $31.6 \%$ indicam trabalhar em organizações com cerca de 10 a 50 colaboradores, seguindo-se-lhes os grupos de respondentes cujas organizações possuem dimensões que variam entre os 51 e os 250 colaboradores $(22.1 \%)$. O Quadro 1 sumariza as características da amostra utilizada neste estudo. 
Quadro 1. Características da amostra

\begin{tabular}{|c|c|c|c|c|}
\hline & M & DP & $\mathrm{n}$ & $\%$ \\
\hline \multicolumn{5}{|l|}{ Sexo } \\
\hline Masculino & & & 174 & 42.6 \\
\hline Feminino & & & 233 & 57.1 \\
\hline Idade & 39.85 & 11.77 & & \\
\hline \multicolumn{5}{|l|}{ Habilitações } \\
\hline Sabe ler e escrever, sem possuir o $1^{\circ}$ Ciclo do Ensino Básico & & & 6 & 1.5 \\
\hline $1^{\text {o }}$ ciclo do ensino básico (primária) & & & 7 & 1.7 \\
\hline $2^{\circ}$ ciclo do ensino básico $\left(6 .^{\circ}\right.$ ano $)$ & & & 28 & 6.9 \\
\hline $3^{\circ}$ ciclo do ensino básico $\left(9 .^{\circ}\right.$ ano $)$ & & & 71 & 17.4 \\
\hline Ensino Secundário (12. ${ }^{\circ}$ ano) & & & 155 & 38 \\
\hline Bacharelato & & & 6 & 1.5 \\
\hline Licenciatura em curso & & & 12 & 2.9 \\
\hline Pós-Graduação/Mestrado/Licenciatura Pré Bolonha & & & 77 & 18.9 \\
\hline Licenciatura Pós Bolonha & & & 37 & 9.1 \\
\hline Mestrado Pré Bolonha & & & 3 & .7 \\
\hline Doutoramento & & & 4 & 1 \\
\hline \multicolumn{5}{|l|}{ Situação Profissional } \\
\hline Trabalhador do Estado & & & 124 & 30.4 \\
\hline Trabalhador no setor privado & & & 282 & 69.1 \\
\hline \multicolumn{5}{|l|}{ Vínculo com a Organização } \\
\hline Prestador de serviços (recibos verdes) & & & 22 & 5.4 \\
\hline Contrato a termo & & & 127 & 31.1 \\
\hline Contrato sem termo & & & 254 & 62.3 \\
\hline Tempo de trabalho na organização & 11.46 & 10.48 & & \\
\hline \multicolumn{5}{|l|}{ Tempo com o superior hierárquico } \\
\hline 3 meses & & & 12 & 2.9 \\
\hline Entre 3 e 6 meses & & & 26 & 6.4 \\
\hline Entre 6 meses e 1 ano & & & 67 & 16.4 \\
\hline \multirow[t]{2}{*}{ Mais de 1 ano } & & & 298 & 73 \\
\hline & & & $\mathrm{N}$ & $\%$ \\
\hline \multicolumn{5}{|l|}{ Desempenho de funções de chefia } \\
\hline Sim & & & 94 & 23 \\
\hline Não & & & 311 & 76.2 \\
\hline \multicolumn{5}{|l|}{ Dimensão da organização } \\
\hline Até 9 colaboradores & & & 69 & 16.9 \\
\hline Entre 10 e 50 colaboradores & & & 129 & 31.6 \\
\hline Entre 51 e 250 colaboradores & & & 90 & 22.1 \\
\hline Entre 251 e 500 colaboradores & & & 33 & 8.1 \\
\hline Entre 501 e 1000 colaboradores & & & 26 & 6.4 \\
\hline Mais de 1001 colaboradores & & & 54 & 13.2 \\
\hline \multicolumn{5}{|l|}{ Setor de atividade da Organização } \\
\hline Indústria Transformadora & & & 59 & 14.5 \\
\hline Indústria Extrativa & & & 6 & 1.5 \\
\hline Comércio por grosso e a retalho & & & 47 & 11.5 \\
\hline Alojamento e restauração & & & 30 & 7.4 \\
\hline Agricultura, pecuária, pescas & & & 14 & 3.4 \\
\hline Construção & & & 8 & 2 \\
\hline Produção e distribuição de eletricidade, gás e água & & & 9 & 2.2 \\
\hline Transportes e armazenagem & & & 14 & 3.4 \\
\hline Educação e ciência & & & 77 & 18.9 \\
\hline Saúde humana e apoio social & & & 57 & 14 \\
\hline Atividades imobiliárias, alugueres e serviços prestados às empresas & & & 6 & 1.5 \\
\hline Artes e indústrias criativas & & & 5 & 1.2 \\
\hline Tecnologia de informação e comunicações & & & 14 & 3.4 \\
\hline Outra & & & 59 & 14.5 \\
\hline \multicolumn{5}{|l|}{ Salário auferido } \\
\hline Até $500 €$ & & & 58 & 14.2 \\
\hline Entre $501-1000 €$ & & & 243 & 59.6 \\
\hline Entre $1001-1500 €$ & & & 71 & 17.4 \\
\hline Entre $1501-2000 €$ & & & 15 & 3.7 \\
\hline Entre $2001-2500 €$ & & & 3 & .7 \\
\hline Entre $2501-3000 €$ & & & 6 & 1.5 \\
\hline Entre 3001-3500€ & & & 1 & .2 \\
\hline
\end{tabular}

Nota. Média $(M)$; Desvio-Padrão $(D P)$; o quadro não inclui a indicação dos 'missing values' (não respostas)

Procedimento de recolha de dados e critérios éticos

$\mathrm{Na}$ recolha de dados todos os procedimentos formais e éticos inerentes a uma investigação desta natureza foram assegurados (Ordem dos Psicólogos Portugueses, 2011). Foi apresentado o projeto ao Comité de Ética de uma universidade pública portuguesa, tendo sido emitido um parecer 
favorável. Os dados foram recolhidos por estudantes de Psicologia da referida universidade como parte do processo da sua formação numa unidade curricular de métodos de investigação em psicologia. Cada estudante passou por um cuidadoso treino específico para a realização da tarefa, quer em termos das competências técnicas, quer dos critérios éticos aplicáveis.

Antes do preenchimento dos questionários, foi facultada aos respondentes informação relativa aos objetivos gerais do projeto, à identificação de toda a equipa de investigação, ao tempo estimado para conclusão da tarefa, bem como ao contexto em que a mesma estava a ser realizada. Foram assegurados o anonimato e a confidencialidade das respostas individuais, esclarecendo-se que os resultados seriam apresentados agrupados. Foi solicitada a assinatura de um consentimento informado previamente assinado pelo responsável da equipa de investigação e pelo estudante aplicador. $\mathrm{O}$ consentimento informado indicava $\mathrm{o}$ compromisso de cada parte perante a tarefa. Foi ainda dada a possibilidade de os respondentes indicarem numa folha à parte o seu endereço de correio eletrónico para receberem um resumo dos resultados finais. Cada estudante distribuiu três questionários a colaboradores de organizações de várias zonas de Portugal, no âmbito da sua rede de relações, com as mais diversas ocupações profissionais, de forma a obter-se um $N$ elevado e diverso. Após a aplicação, cada estudante apresentou um relatório sobre a tarefa realizada e assinou um termo de responsabilidade.

\section{Instrumentos}

\section{Toxic Leadership Scale}

A Toxic Leadership Scale (TLS; Schmidt, 2008) não dispunha previamente de uma versão portuguesa. Foram, portanto, realizados no presente estudo os procedimentos necessários para o seu desenvolvimento e validação. Após solicitada ao seu autor permissão para utilização da escala - que foi concedida -, realizou-se a tradução dos itens por três peritos de Psicologia do Trabalho e das Organizações proficientes em inglês técnico, que realizaram uma primeira tradução para o português, separadamente. Seguidamente, reuniram-se as três traduções e consolidou-se uma versão em língua portuguesa, que foi revista e ajustada com apoio de um psicólogo do trabalho e das organizações bilingue. Posteriormente, a versão consolidada foi submetida a uma retroversão por um tradutor profissional de língua inglesa, cuja língua materna é o inglês, vivendo em Portugal há mais de 20 anos. A comparação do original com a retroversão permitiu a obtenção de uma versão em língua portuguesa com pequenos ajustamentos de linguagem. Por fim, foi realizada uma reflexão falada sobre os itens já traduzidos, com três indivíduos, distintos em idade, qualificações e situação profissional, enquadrados nos critérios de elegibilidade dos participantes. Este processo conduziu à versão final consolidada da escala em língua portuguesa (ver Anexo A).

A resposta aos itens da TLS utiliza uma escala de avaliação do tipo Likert, de seis pontos, variando de "Discordo totalmente" ( $1=$ o meu líder não é nada assim) a "Concordo totalmente" ( $6=0$ meu líder é mesmo assim). A versão original da TLS é composta por cinco fatores: (1) Supervisão Abusiva, com sete itens, referindo-se a comportamentos hostis, quer verbais quer nãoverbais, do líder para com os seus subordinados (e.g., "Ridiculariza os subordinados"); (2) Liderança Autoritária' (seis itens), referindo-se aos comportamentos do líder que restringem a autonomia e a capacidade de iniciativa dos seus subordinados (e.g., "Controla o modo como os subordinados realizam as suas tarefas"); (3) Narcisismo (cinco itens), referindo-se ao facto do líder ter uma autoimagem de grande imponência, uma inabilidade para mostrar empatia para com outros, desprezando as aptidões e os esforços dos outros (e.g., "Sente-se com direitos especiais"); (4) Autopromoção (cinco itens), referindo-se a comportamentos por parte do líder que apenas atendem aos seus próprios interesses, depreciando comportamento de rivais ou talentos de subordinados (e.g., "Só oferece ajuda às pessoas que the possam trazer vantagens"); e, (5) Imprevisibilidade (sete itens), referindo-se a comportamentos imprevisíveis por parte do líder, quer por mudanças de humor, quer por outras, fazendo assim com que os subordinados não saibam com o que contar (e.g., "Expressa raiva aos subordinados sem razão aparente"). 


\section{Empowering Leadership Questionnaire}

O Empowering Leadership Questionnaire (ELQ; Arnold, Turner, Barling, Kelloway, \& McKee, 2007) havia já sido aplicado em Portugal numa amostra de 315 sujeitos (Serrano, 2015), tendo sido realizados previamente todos os procedimentos de tradução, retroversão e reflexão falada. A permissão de utilização do questionário foi também obtida junto do respetivo autor principal.

Este instrumento constitui uma operacionalização do constructo de liderança empoderadora. Os respondentes revelam a sua perceção quanto aos comportamentos dos seus líderes utilizando uma escala de avaliação de tipo Likert, de cinco pontos, que varia entre "Nunca" (1=o líder nunca se comporta assim) e "Sempre" (5=o líder comporta-se sempre assim). É composto por 38 itens agrupados em cinco fatores: (1) Liderar pelo exemplo, composto por cinco itens, referindo-se a comportamentos que mostram o comprometimento do líder para com o seu trabalho e o trabalho dos membros da sua equipa (e.g., "Estabelece elevados padrões de desempenho pelo seu próprio comportamento"); (2) Participação nas tomadas de decisão (seis itens), referindo-se ao uso que o líder dá às informações obtidas pelos membros da equipa e à relevância que o mesmo dá às opiniões da sua equipa na tomada de decisão (e.g., "Incentiva os membros do grupo (departamento, secção) a expressar ideias/sugestões"; (3) Coaching (11 itens), referindo-se a um conjunto de comportamentos por parte do líder para ensinar os membros da equipa e ajudá-los a tornarem-se autossuficientes (e.g., "Ajuda-nos a ver áreas em que precisamos de mais formação"); (4) Informar (seis itens), referindo-se à divulgação, pelo líder, de diversas informações sobre a empresa, como a missão e filosofia, ou outras informações importantes (e.g., "Explica os objetivos da organização"); e, por fim, (5) Mostrar preocupação/Interagir com o grupo (10 itens), referindo-se a comportamentos por parte do líder que demonstram preocupação pelo bem-estar dos membros da equipa, preocupando-se com o que estes estão a fazer no momento, trabalhando em conjunto com estes, etc. (e.g., "Demonstra preocupação pelo sucesso dos membros do grupo de trabalho (departamento, secção). Esta escala dispõe de um item inverso no fator 2 (item 11). A escala é apresentada no Anexo B.

\section{Análise de dados}

Para o registo e processamento de dados foi utilizada a versão 22.0 dos softwares IBM SPSS Statistics (IBM Corp. Released 2013) e IBM SPSS AMOS (Arbuckle, 2013). Os missing- values, all MCAR (completely at random) e inferiores a $2 \%$ foram substituídos pelo método Series mean. A validade fatorial das escalas foi avaliada com análises fatoriais confirmatórias através do AMOS, método de estimação da máxima verosimilhança (Jöreskog \& Sörbom, 2004). A existência de outliers foi analisada pela distância quadrática de Mahalanobis (Tabachnick \& Fidell, 2013).

A normalidade das variáveis foi avaliada pelos coeficientes de assimetria $(S k)$ e a curtose $(K u)$ de todos os itens, que não mostraram valores que se afastassem excessivamente dos valores considerados ajustados para a assunção do pressuposto da normalidade (Finney \& Distefano, 2006; Kline, 2011; Marôco, 2014), dado terem sido obtidos valores de $|s k|<2$ e de $|k u|<6$.

Considerando que as medidas TLS e ELQ foram previamente validadas em língua inglesa, considerou-se adequada a realização de análises fatoriais confirmatórias (AFC). A qualidade do ajustamento do modelo fatorial foi avaliada pelos índices $\chi^{2} / \mathrm{df}$ (chi-square/degrees of freedom; ajustamento aceitável $\leq 5$; bom ajustamento $\leq 2$; Arbuckle, 2013; Schumacker \& Lomax, 2012), NFI (normed fit index; bom ajustamento > .80; Schumacker \& Lomax, 2012), CFI (comparative fit index; bom ajustamento > .90; Bentler, 1990), SRMR (standardized root mean square residual; ajustamento apropriado <.08; Brown, 2015), TLI (Tucker-Lewis index; ajustamento apropriado > .90; Brown, 2015) e RMSEA (root mean square error of approximation; bom ajustamento <.05, ajustamento aceitável <.08; Kline, 2011; Schumacker \& Lomax, 2012), comparando com os valores indicativos de um ajustamento bom ou aceitável na literatura. A consistência interna foi estimada pelo coeficiente alfa de Cronbach (Cronbach, 1951). A precisão compósita e a variância média extraída de cada um dos fatores foram avaliadas como descrito em Fornell e Larcker (1981). Por último, foi utilizada uma análise descritiva e analisadas as intercorrelações 
entre os fatores das duas escalas. A magnitude dos coeficientes de correlação foi interpretada de acordo com Cohen (1988).

\section{Resultados}

Realizou-se uma AFC através do programa AMOS à estrutura de cinco fatores do Empowering Leadership Questionnaire (Arnold et al., 2007). Os índices de ajustamento do primeiro modelo revelaram coeficientes de $\chi^{2} / \mathrm{df}=3.28$, $\mathrm{CFI}=.907, \mathrm{NFI}=.871, \mathrm{TLI}=.900, \quad \mathrm{SRMR}=.037 \mathrm{e}$ RMSEA $=.075, p<.001$. Este modelo mostrou um bom ajustamento considerando todos os índices de ajustamento à exceção do $\chi 2 / \mathrm{df}$ e do RMSEA, que mostraram ser aceitáveis. A representação do modelo estrutural estimado consta na Figura 1.

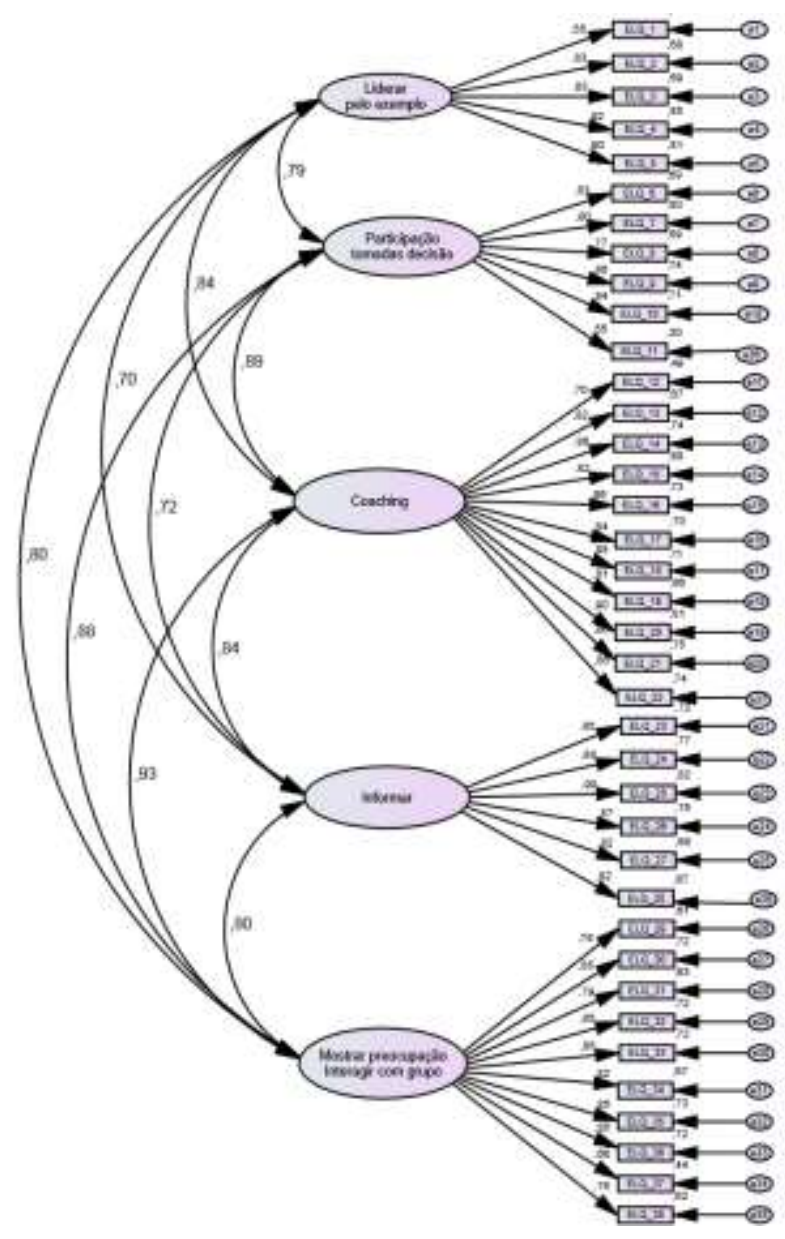

Figura 1. Análise fatorial confirmatória do Empowering Leadership Questionnaire (ELQ): coeficientes de regressão estandardizados e proporções de variância explicada $\left(\mathrm{R}^{2}\right)$
Em seguida, realizámos uma AFC à estrutura penta-fatorial da escala TLS. Os índices de ajustamento indicaram que o modelo é bem ajustado considerando os índices CFI=.931, $\mathrm{NFI}=.898$, TLI=.924 e SRMR=.041. O modelo evidenciou um ajustamento aceitável atendendo ao $\chi^{2} / \mathrm{df}=2.84$ e ao RMSEA=.067 (cf. modelo estrutural estimado na Figura 2).

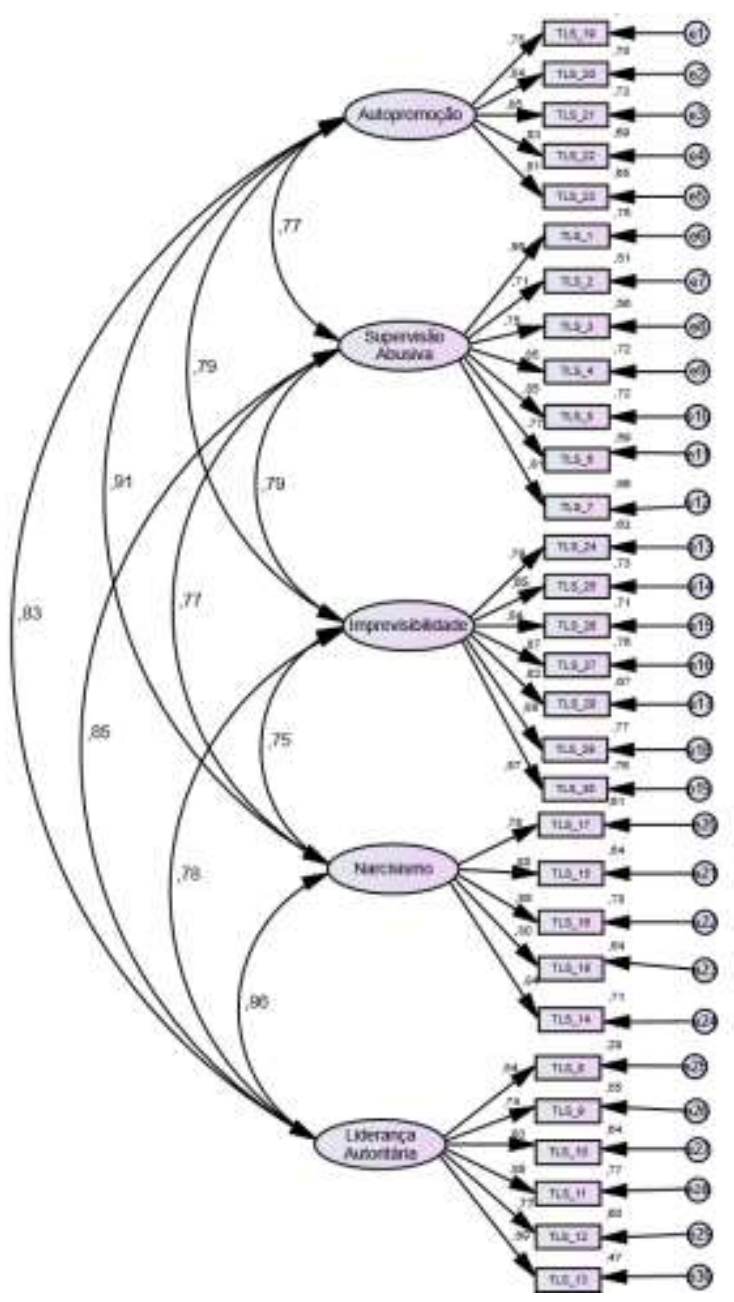

Figura 2. Análise fatorial confirmatória da Toxic Leadership Scale (TLS): coeficientes de regressão estandardizados e proporções de variância explicada $\left(\mathrm{R}^{2}\right)$

No Quadro 2 podem consultar-se as estatísticas descritivas, os coeficientes de precisão compósita, variância extraída, consistência interna e matriz de intercorrelações das duas medidas em análise (ELQ e TLS). Considerando a medida de liderança empoderadora, os fatores que evidenciam um resultado médio mais elevado são o F5 - Mostrar Preocupação $(M=3.46)$ e o $F 1$ Liderança pelo exemplo $(M=3.45)$, respeitando o resultado mais baixo ao $F 4$ - Informação $(M=2.73)$. 
Quadro 2. Descritivas, Precisão compósita, variância extraída, coeficientes de consistência interna e matriz de intercorrelações das medidas ELQ e TLS

\begin{tabular}{|c|c|c|c|c|c|c|c|c|c|c|c|c|c|c|c|c|}
\hline & Min & $\operatorname{Max}$ & $M$ & $D P$ & $P C$ & $V E$ & $\begin{array}{c}\text { ELQ } \\
\text { F1 }\end{array}$ & $\begin{array}{c}\text { ELQ } \\
\text { F2 }\end{array}$ & $\begin{array}{c}\text { ELQ } \\
\text { F3 }\end{array}$ & $\begin{array}{c}\text { ELQ } \\
\text { F4 }\end{array}$ & $\begin{array}{l}\text { ELQ } \\
\text { F5 }\end{array}$ & $\begin{array}{c}\text { TLS } \\
\text { F1 }\end{array}$ & $\begin{array}{c}\text { TLS } \\
\text { F2 }\end{array}$ & $\begin{array}{l}\text { TLS } \\
\text { F3 }\end{array}$ & $\begin{array}{c}\text { TLS } \\
\text { F4 }\end{array}$ & $\begin{array}{c}\text { TLS } \\
\text { F5 }\end{array}$ \\
\hline $\begin{array}{l}\text { ELQ F1: Liderança } \\
\text { pelo exemplo }\end{array}$ & 1 & 5 & 3.45 & 1.07 & .91 & .67 & $(.91)$ & $.71 * *$ & $.77 * *$ & $.64 * *$ & $.72 * *$ & $-.52 * *$ & $-.46^{* *}$ & $-.51 * *$ & $-.57 * *$ & $-.47 * *$ \\
\hline $\begin{array}{l}\text { ELQ F2: Tomada de } \\
\text { decisão }\end{array}$ & 1 & 5 & 3.30 & .96 & .91 & .64 & & $(.91)$ & $.84 * *$ & $.68 * *$ & $.82 * *$ & $-.58 * *$ & $-.61 * *$ & $-.56 * *$ & $-.60 * *$ & $-.54 * *$ \\
\hline ELQ F3: Coaching & 1 & 5 & 3.35 & 1.01 & .96 & .70 & & & $(.96)$ & $.80 * *$ & $.88 * *$ & $-.55 * *$ & $-.52 * *$ & $-.50 * *$ & $-.59 * *$ & $-.48 * *$ \\
\hline ELQ F4: Informação & 1 & 5 & 2.73 & 1.34 & .94 & .73 & & & & $(.94)$ & $.77 * *$ & $-.45 * *$ & $-.42 * *$ & $-.41 * *$ & $-.50 * *$ & $.41 * *$ \\
\hline $\begin{array}{l}\text { ELQ F5: Mostrar } \\
\text { preocupação }\end{array}$ & 1 & 5 & 3.46 & .98 & .95 & .66 & & & & & $(.95)$ & $-.60 * *$ & $-.57 * *$ & $-.54 * *$ & $-.64 * *$ & $.54 * *$ \\
\hline $\begin{array}{l}\text { TLS F1: Supervisão } \\
\text { abusiva }\end{array}$ & 1 & 6 & 2.28 & 1.21 & .93 & .64 & & & & & & $(.87)$ & $.78^{* *}$ & $.72 * *$ & $.72 * *$ & $.75^{* *}$ \\
\hline $\begin{array}{l}\text { TLS F2: Liderança } \\
\text { Autoritária }\end{array}$ & 1 & 6 & 2.65 & 1.16 & .88 & .55 & & & & & & & $(.92)$ & $.77 * *$ & $.73 * *$ & $.72 * *$ \\
\hline TLS F3: Narcisismo & 1 & 6 & 3.39 & 1.05 & .91 & .68 & & & & & & & & $(.91)$ & $.82 * *$ & $.69 * *$ \\
\hline $\begin{array}{l}\text { TLS F4: Auto- } \\
\text { promoção }\end{array}$ & 1 & 6 & 2.49 & 1.33 & .91 & .67 & & & & & & & & & $(.91)$ & $.73 * *$ \\
\hline $\begin{array}{l}\text { TLS F5: } \\
\text { Imprevisibilidade }\end{array}$ & 1 & 6 & 2.72 & 1.40 & .95 & .72 & & & & & & & & & & $(.95)$ \\
\hline
\end{tabular}

Na medida de liderança tóxica, o fator que mostra um resultado médio mais elevado é o $F 3$ Narcisismo $(M=3.39)$, ao passo que o mais baixo respeita ao $F 1$ - Supervisão abusiva $(M=2.28)$. Conforme pode ser visto na diagonal da matriz da Quadro 2, todos os fatores das escalas apresentam uma boa consistência interna $(\alpha \geq .87)$. Os índices de precisão compósita são aceitáveis, visto serem iguais ou superiores a .70 (Hair, Anderson, Tatham, \& Black, 2008). Na variância média extraída, todos os fatores se situaram acima de .50 , coeficiente a partir do qual Bagozzi e Yi (1988) consideram uma variância extraída aceitável.

A matriz de intercorrelações entre os dois tipos de liderança em estudo aponta para relações negativas de magnitudes moderada $(.30 \leq|r|<.50) \mathrm{e}$ elevada ( $|r| \geq .50)$ (Cohen, 1988), suportando a hipótese formulada.

\section{Discussão}

Tal como mencionado anteriormente, este trabalho teve como objetivo realizar a validação recíproca da versão portuguesa da Toxic Leadership Scale, e do Empowering Leadership Questionnaire. Os resultados mostram que a estrutura fatorial proposta possui um bom ajustamento. As dimensões de ambas as escalas correlacionam-se negativamente em magnitude moderada ou elevada, evidenciando a existência de validade divergente. Tal pode ser interpretado como indicador de que se trata de dois constructos que, sendo conceptualmente antagónicos, não o são de tal forma que tornem a sua utilização redundante. Efetivamente, nenhuma das correlações encontradas é muito forte.

Perceber e medir estes constructos torna-se crítico para perceber o sucesso de uma organização (Parris \& Welty-Peachey, 2013). Com efeito, estes são constructos que, tendo efeitos detrimentais ou desenvolvimentais nos seus colaboradores, expressam fenómenos que impactam indiretamente nos resultados organizacionais que esses mesmos colaboradores concretizam. As análises realizadas revelam que ambos os instrumentos possuem boas qualidades psicométricas e boa consistência interna. Verificou-se que se correlacionam negativamente, o que vai ao encontro do esperado, podendo assim reforçar a validação mútua das escalas.

A liderança é um dos conceitos mais estudados ao longo do tempo no âmbito da Psicologia das Organizações. O presente trabalho apresenta um contributo particular e significativo para o estudo deste tema em países de língua portuguesa, na medida em que disponibiliza a versão da TLS nesta língua, permite o estudo da 
liderança (TLS e ELQ) por dimensões, e apresenta estudos de validação de ambas as escalas para a população portuguesa, o que constitui um ponto de partida importante para estudos semelhantes noutros países lusófonos.

\section{Referências}

Arbuckle, J. L. (2013). Amos 22 user's guide. Chicago, IL: SPSS.

Arnold, K. A., Turner, N., Barling, J., Kelloway, E. K., \& McKee, M. C. (2007). Transformational leadership and psychological well-being: The mediating role of meaningful work. Journal of Occupational Health Psychology, 12(3), 193-203.

Auh, S., Menguc, B., \& Jung, Y. S. (2014). Unpacking the relationship between empowering leadership and service-oriented citizenship behaviors: A multilevel approach. Journal of the Academy of Marketing Science, 42(5), 558-579.

Bagozzi, R., \& Yi, Y. (1988). On the evaluation of structural equation models. Journal of the Academy of Marketing Science, 16(1), 74-94.

Bambale, A. (2014). Relationship between servant leadership and organizational citizenship behaviors: Review of literature and future research directions. Journal of Marketing and Management, 5(1), 1-16.

Bentler, P. M. (1990). Comparative fit indexes in structural models. Psychological Bulletin, 107(2), 238-246.

Brown, T. (2015). Confirmatory factor analysis for applied research. New York: The Guilford Press.

Chen, G., Sharma, P. N., Edinger, S. K., Shapiro, D. L., \& Farh, J.-L. (2011). Motivating and demotivating forces in teams: Cross-level influences of empowering leadership and relationship conflict. Journal of Applied Psychology, 96(3), 541-557.

Cohen, J. (1988). Statistical power analysis for the behavioral sciences (2nd ed.). New York: Academic Press.

Cronbach, L. J. (1951). Coefficient Alpha and the internal structure of tests. Psychometrika, 16(3), 297-334.
Di Genio, J. (2002). The toxic boss. The Armed Forces, Comptroller 47(1), 14-18.

Dyck, D. (2001). The toxic workplace. Benefits Canada, 25(3), 52-57.

Finney, S. J., \& Distefano, C. (2006). Non-normal and categorical data in structural equation modeling. In G. R. Hancock \& R. O. Mueller (Eds.), Structural equation modeling: A second course (pp. 269-314). Greenwich, CT: Information Age Publishing.

Flynn, G. (1999). Stop toxic leaders before they stop you! Workforce, 78(8), 44-46.

Fornell, C., \& Larcker, D. F. (1981). Evaluating structural equation models with unobservable variables and measurement error. Journal of Marketing Research, 18(1), 39-50.

Hair, J. F., Anderson, R. E., Tatham, R. L., \& Black, W. C. (2008). Multivariate data analysis (7th ed.). Pearson Prentice-Hall.

Hansbrough, T. \& Jones, G. E. (2014). Inside the minds of narcissists: How narcissistic leaders' cognitive processes contribute to abusive supervision. Zeitschrift für Psychologie, 222(4), 214-220.

Hernandez, M., Eberly, M. B., Avolio, B. J., \& Johnson, M. D. (2011). The loci and mechanisms of leadership: Exploring a more comprehensive view of leadership theory. The Leadership Quarterly, 22, 1165-1185.

Hill, M. M., \& Hill, A. (2008). Investigação por questionário. ( $2^{\mathrm{a}}$ ed.) Lisboa: Edições Sílabo.

IBM Corp. Released (2013). IBM SPSS Statistics for Windows, Version 22.0. Armonk, NY: IBM Corp.

Jago, A.G. (1982). Leadership: Perspectives in theory and research. Journal of management science, 28(3), 315-332.

Jöreskog, K. G., \& Sörbom, D. (2004). LISREL 8.7 for Windows [Computer Software]. Lincolnwood, IL: Scientific Software International, Inc.

Kline, R. B. (2011). Principles and practice of structural equation modeling (3rd ed.). New York: The Guilford Press.

Kusy, M., \& Holloway, E. (2009). Toxic workplace! Managing toxic personalities and their systems of power. San Francisco: JosseyBass.

Lipman-Blumen, J. (2005). The allure of toxic leaders: Why we follow destructive bosses and 
corrupt politicians - and how we can survive them. Oxford: Oxford University Press.

Lorinkova, N. M., Pearsall, M. J., \& Sims, H. P. (2013). Examining the diferencial longitudinal performance of directive versus empowering leadership teams. Academy of Management Journal, 56(2), 573-596.

Macklem, K. (2005). The toxic workplace. Maclean's, 118(5), 34-35.

Marôco, J. (2014). Análise estatística com o SPSS Statistics ( $6^{\mathrm{a}}$ ed). Pêro Pinheiro: Report Number.

May, D., Wesche, J., Heinitz, K., \& Kerschreiter, R. (2014). Coping with destructive leadership: Putting forward an integrated theoretical framework for the interaction process between leaders and followers. Zeitschrift für Psychologie, 222(4), 203-213.

Miniotaité, A., \& Bučiūnienè, I. (2013). Explaining authentic leadership work outcomes from the perspective of selfdetermination theory. Organizaciju vadyba: Sisteminiai Tyrimai, 65, 63-75.

Ordem dos Psicólogos Portugueses (2011). Código deontológico. Acedido em https://www.ordemdospsicologos.pt/ficheiros/ documentos/web_cod_deontologico_pt_revisa o_2016.pdf

Parris, D., L., \& Welty-Peachey, J. (2013). A systematic literature review of servant leadership theory in organizational contexts. Journal of Business Ethics, 113(3), 377-393.

Pelletier, K. (2010). Leader toxicity: An empirical investigation of toxic behavior and rhetoric. Leadership, 6(4), 373-389.

Reed, G. (2004). Toxic leadership. Military Review, July-August, 67-71.

Salessi, S., \& Omar, A. (2016). Desarrollo y Validación de una Escala para Medir Actuación Emocional en el Trabajo - Development and validation of a scale to measure emotional display rule at work. Revista Iberoamericana de Diagnóstico y Evaluación - e Avaliação Psicológica, 41(1), 66-79.

Schmidt, A. (2008). Development and validation of the toxic leadership scale (master thesis). Acedido em Digital Repository at the University of Maryland ( $\mathrm{n}^{\circ} 5358$ ).

Schumacker, R. E. \& Lomax, R. G. (2012). A beginner's guide to structural equation modeling. New York: Routledge.
Serrano, J. (2015). As componentes educativa e cultural das organizações como fatores incrementais da cooperação interinstitucional $e \quad d o$ desenvolvimento organizacional (dissertação de doutoramento). Acedido em Repositório Universidade de Évora, http://hdl.handle.net/10174/16233

Smircich, L., \& Morgan, G. (1982). Leadership: The management of meaning. Journal of Applied Behavioral Science, 18(3), 257-273. https://doi.org/10.1177/002188638201800303

Solano, A. C., \& Minervino, R. (2007). Motivación para liderar, inteligencia práctica y efectividad de los líderes - Motivation to lead practical intelligence and leaders' effectiveness. Revista Iberoamericana de Diagnóstico y Evaluación - e Avaliação Psicológica, 23(1), 175-199.

Srivastava, A., Bartol, K. M., \& Locke, E. A. (2006). Empowering leadership in management teams: Effects on knowledge sharing, efficacy, and performance. Academy of Management Journal, 49(6), 1239-1251.

Steele, J. (2011). Antecedents and consequences of toxic leadership in the U.S. Army: A twoyear review and recommended solutions (Technical Report 2011-3). Kansas: Center for Army Leadership.

Tabachnick, B. G., \& Fidell, L. S. (2013). Using multivariable statistics (6th ed.). California: Allyn \& Bacon.

Tuckey, M. R., Dollard, M. F., \& Bakker, A. B. (2012). Empowering leaders optimize working conditions for engagement: a multilevel study. Journal of Occupational Health Psychology, 17(1),15-27.

Walton, M. (2007). Leadership toxicity: an inevitable affliction of organizations? Organizations \& People, 14(1), 19-27.

Whicker, M. L. (1996). Toxic leaders: when organizations go bad. Westport, CT: Quorum Books.

Williams, D. F. (2005). Toxic leadership in the U.S. Army (Strategy research project). Pennsylvania: U.S. Army War College.

Wilson-Starks, K. Y. (2003). Toxic leadership. Trans leadership, Inc. Acedido em http://transleadership.com/wpcontent/uploads/ToxicLeadership.pdf 


\section{Anexo A.}

Toxic Leadership Scale (Schmidt, 2008) - itens da Versão Portuguesa

Responda em todas as afirmações considerando a seguinte questão:

O/A meu/minha superior(a) hierárquico(a)/chefe:

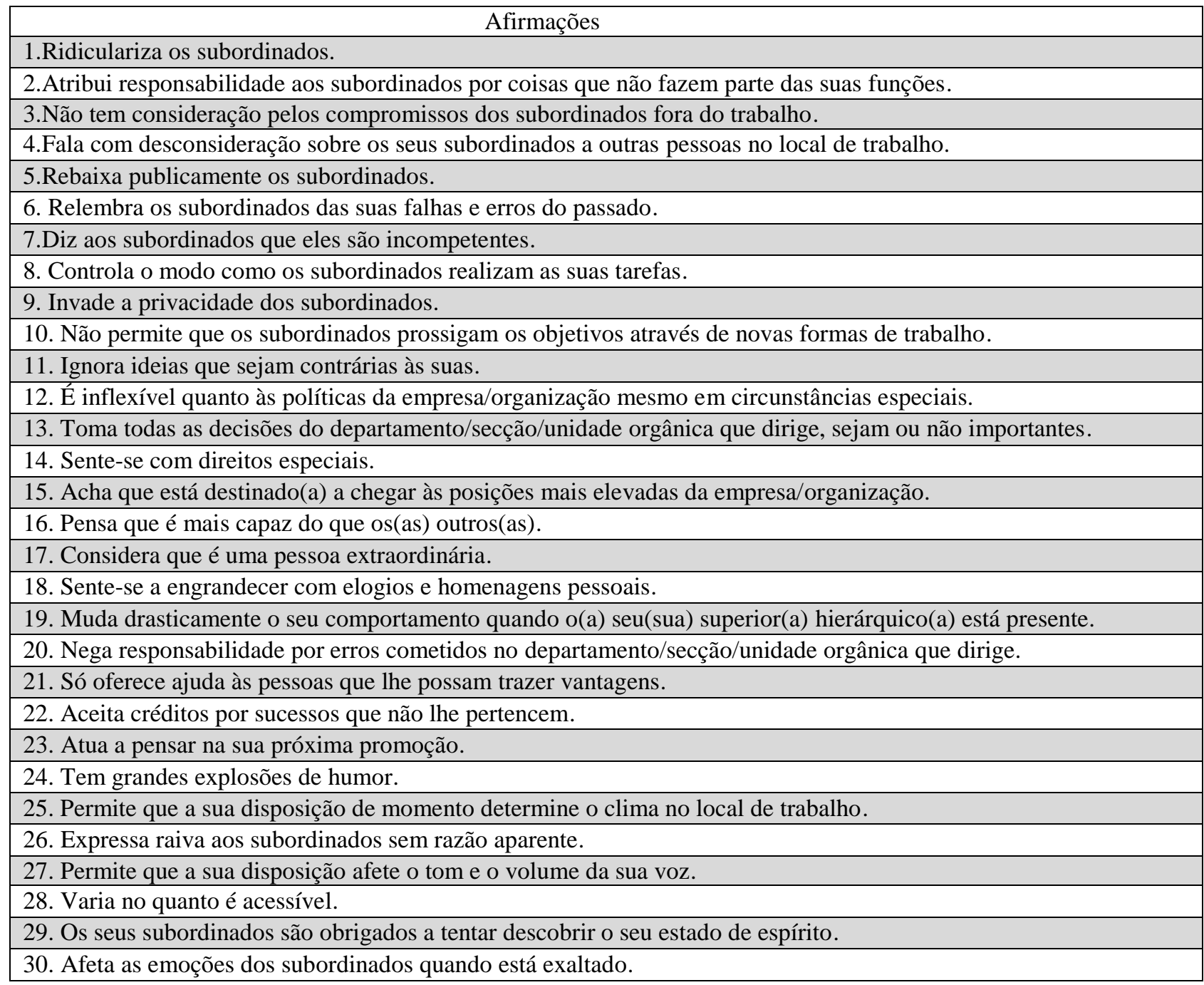




\section{Anexo B.}

Empowering Leadership Questionnaire (Arnold, Arad, Rhoades, \& Drasgow, 2000) - itens da Versão Portuguesa

Responda em todas as afirmações considerando a seguinte questão:

O/A meu/minha superior(a) hierárquico(a)/chefe:

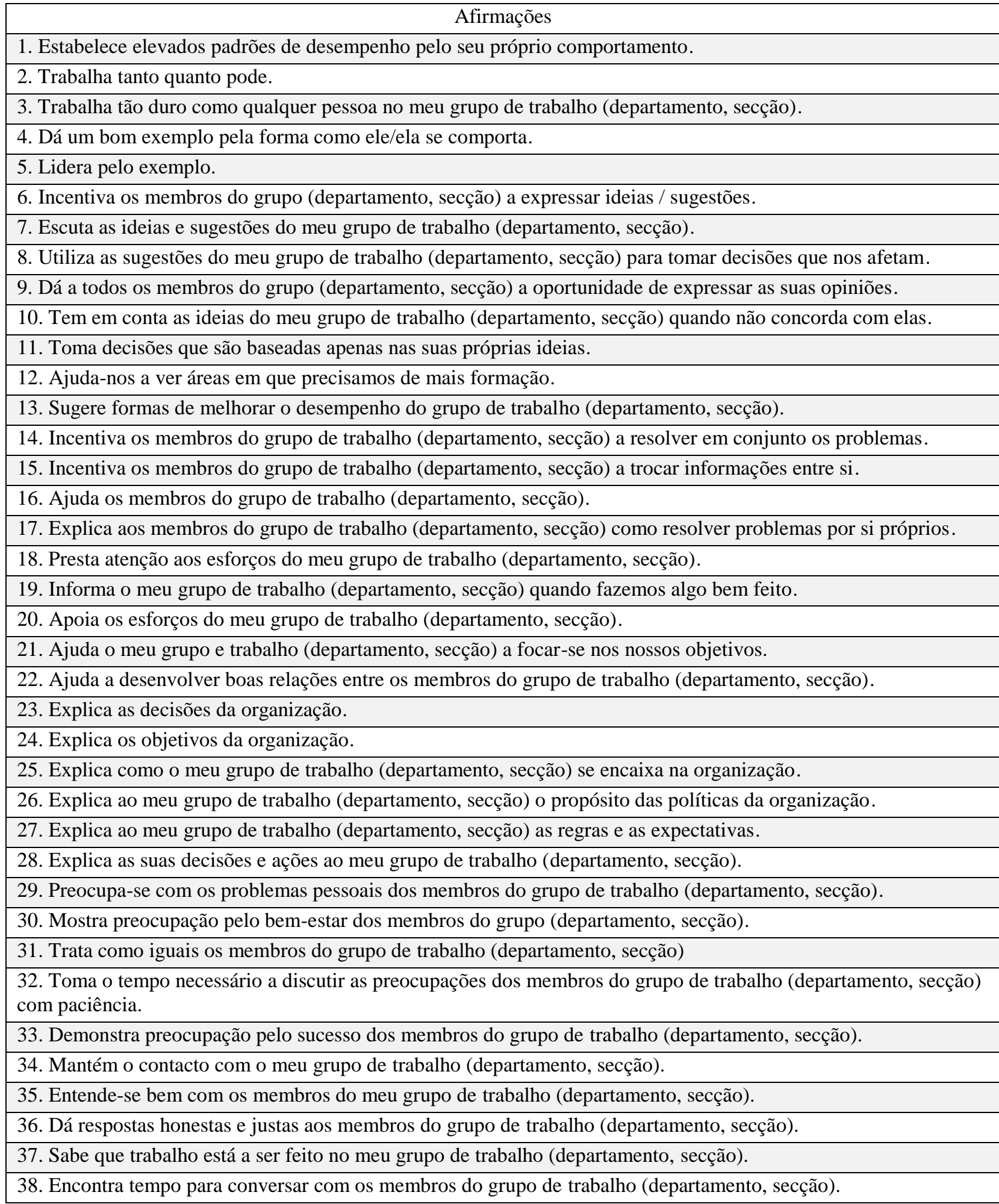

